\title{
AP-VAS 2012 case report: a case of lupus nephritis with predominant synchronous cellular crescent formation and myeloperoxidase-antineutrophil cytoplasmic antibody seropositivity
}

\author{
Tetsuya Makiishi • Tomoyuki Shirase • \\ Shinya Yamamoto $\cdot$ Sayako Maeda
}

Received: 28 August 2012/ Accepted: 17 October 2012/Published online: 30 January 2013

(C) Japanese Society of Nephrology 2012

\begin{abstract}
Several cases with an overlap of antineutrophil cytoplasmic antibody (ANCA)-associated glomerulonephritis (GN) and lupus nephritis (LN) features have been reported in recent years. However, the clinical and the pathologic features of this condition, including mode of development, histology, and response to treatment, are not fully understood. We report a 77-year-old woman who was diagnosed with Sjögren syndrome 15 years previously. The patient presented with acute worsening of renal function and was diagnosed with new-onset systemic lupus erythematosus. A renal biopsy specimen revealed proliferative LN with synchronous cellular crescents. She was also seropositive for myeloperoxidase-ANCA. Together with the positive staining for immunoglobulins and complement factors on immunofluorescence microscopy and scant subendothelial deposits by electron microscopy, we reached a diagnosis of ANCA-associated crescentic GN overlapping with LN. Although immunosuppressive treatment with methylprednisolone pulse therapy and intravenous cyclophosphamide followed by oral predonisolone was initiated, along with intermittent hemodialysis, these treatments did not induce remission of her GN. Therefore,
\end{abstract}

T. Makiishi $(\varangle) \cdot$ S. Maeda

Division of Nephrology, Department of Internal Medicine,

Otsu Red Cross Hospital, 1-1-35 Nagara, Otsu, Shiga 520-8511,

Japan

e-mail: makiishi@otsu.jrc.or.jp

T. Shirase

Department of Pathology, Otsu Red Cross Hospital,

1-1-35 Nagara, Otsu, Shiga 520-8511, Japan

S. Yamamoto

Division of Nephrology, Department of Internal Medicine,

Kitano Hospital, The Tazuke Kofukai Medical Research

Institute, 2-4-20 Ohgimachi, Kita-Ku, Osaka 530-8480, Japan she continued regular intermittent hemodialysis. However, she died because of candida pneumonia 4 months after admission. Generally, the glomeruli of patients with ANCA-associated GN exhibit different stages of crescents, namely cellular, fibrocellular, or fibrous. The histologically synchronous crescents in this case indicate that ANCAassociated GN overlapping with $\mathrm{LN}$ can progress more rapidly than that without $\mathrm{LN}$. This overlapping type of GN may be resistant to conventional immunosuppressive therapies.

Keywords Lupus nephritis - Crescentic lupus nephritis · Synchronous crescents - Antineutrophil cytoplasmic antibody $\cdot$ Antineutrophil antibody-associated vasculitis

\section{Introduction}

Lupus nephritis (LN) is a classic immune complex-mediated renal disease. The patterns of glomerulonephritis (GN) reflect the sites of immune complex deposition. Renal involvement of antineutrophil antibody-associated vasculitis (AAV) or antineutrophil cytoplasmic antibody (ANCA)-associated GN is histologically characterized by "pauci-immune" necrotizing and crescentic GN. Although both diseases can cause rapid worsening of renal function because of active nephritis, their pathophysiological mechanisms in the development and progression of GN are clearly distinct [1].

Recently, however, several cases with an overlap of both diseases have been reported [2-10]. Their pathologic findings differ from those of typical LN, showing disproportionate necrotizing and crescent formations compared with the degree of subendothelial immune complex deposits and endocapillary proliferation. In addition, the 
ANCA titer, mostly myeloperoxidase (MPO)-ANCA, is elevated. However, the mode of development, histologic features, and response to treatment of this condition are not yet fully understood [2-4]. Here, we describe a patient with new-onset systemic lupus erythematosus (SLE) who experienced acute worsening of renal function. This patient had crescentic LN with MPO-ANCA seropositivity, and her disease was refractory to the treatments used. We also discuss the clinicopathologic features of this disease.

\section{Case report}

A 77-year-old Japanese woman was referred to our hospital because of acute worsening of renal function and general fatigue that persisted for 3 weeks. She regularly attended an outpatient clinic, but was not prescribed any medications based on a previous diagnosis of Sjögren syndrome. Her only symptom had been mild sicca syndrome, which was diagnosed 15 years previously; no other organs were affected. She had been treated for hypertension with olmesartan (20 mg/day) and amlodipine ( $2.5 \mathrm{mg} /$ day) for 3 years. Three months before admission, laboratory tests revealed that her serum creatinine was $0.67 \mathrm{mg} / \mathrm{dl}$ with normal urinalysis. Within 3 months, her serum creatinine had increased to $5.66 \mathrm{mg} / \mathrm{dl}$, and she was referred to our hospital. On admission, her blood pressure was $168 / 72 \mathrm{mmHg}$, pulse rate was $88 / \mathrm{min}$, and temperature was $37.1{ }^{\circ} \mathrm{C}$. Physical examination revealed significant leg edema. Laboratory tests revealed the following values: white blood cell count, $9.4 \times 10^{3} / \mu \mathrm{l}$ (lymphocytes, $\left.1,440 / \mu \mathrm{l}\right)$; red blood cell count, $2.87 \times 10^{6} / \mu \mathrm{l}$, hemoglobin, $8.9 \mathrm{~g} / \mathrm{dl}$; hematocrit, $26.1 \%$; platelet count, $158 \times 10^{3} / \mu \mathrm{l}$; serum creatinine, $7.33 \mathrm{mg} / \mathrm{dl}$; blood urea nitrogen, $87.2 \mathrm{mg} / \mathrm{dl}$; serum albumin, $2.6 \mathrm{~g} / \mathrm{dl}$; C-reactive protein, $1.2 \mathrm{mg} / \mathrm{dl}$; immunoglobulin ( $\mathrm{Ig}) \mathrm{G}$, 2,074 mg/dl; IgA, 195 mg/dl; IgM, 107 mg/dl; complement (C) 3, $44 \mathrm{mg} / \mathrm{dl}$; and $\mathrm{C} 4,10 \mathrm{mg} / \mathrm{dl}$. Urinalysis revealed protein (2+), blood (4+), and 50-100 erythrocytes and 1-4 white blood cells per high-power field. There was no cellular cast in the urine sediment. Urinary protein excretion was $1.84 \mathrm{~g} /$ day. Immunologic studies revealed the following: antinuclear antibody (ANA) titer, 1:1,280 (speckled pattern); anti-dsDNA IgG, $50 \mathrm{IU} / \mathrm{ml}$; anti-ssDNA IgG, $149 \mathrm{AU} / \mathrm{ml}$; anti-ribonucleoprotein antibody, 3,800 IU/ml; anti-Sm antibody, $231 \mathrm{IU} / \mathrm{ml}$; and anti-SS-A antibody, 7,960 IU/ml. The test for anti-SS-B was negative. The MPOANCA titer was elevated (75 EU), while proteinase 3-ANCA and anti-glomerular basement membrane antibody were negative. Chest radiography showed a cardiothoracic ratio of $52 \%$ with no abnormalities. The patient fulfilled 4 (lymphopenia, ANA, DNA antibodies, and proteinuria) of the 11 diagnostic criteria for SLE established by the American College of Rheumatology.
A renal biopsy was performed on day 2 of admission. On light microscopic examination, there were 28 glomeruli within the biopsy. Of these, two were globally sclerotic. All glomeruli showed mild to moderate mesangial hypercellularity (Fig. 1a). Twenty-one glomeruli (75\%) had cellular crescents (Fig. 1b), and some showed degeneration of Bowman's capsule (Fig. 1c). However, there was no evidence of necrosis or endocapillary proliferation. There was diffuse interstitial acute inflammation composed of lymphocytes with mild tubular epithelial changes. Direct immunofluorescence revealed diffuse staining for $\operatorname{IgG}$ in the basement membrane and the mesangium (Fig. 1d), and focal staining for $\mathrm{C} 3$ in the mesangium, whereas staining for $\mathrm{IgA}, \mathrm{IgM}, \mathrm{Clq}$, and $\mathrm{C} 4$ was negative. Electron microscopy showed mesangial and paramesangial deposits with a few subendothelial deposits (Fig. 2). On the basis of these findings, she was diagnosed with ANCA-associated GN overlapping with LN class II [11].

After renal biopsy, intravenous methylprednisolone pulse therapy $(500 \mathrm{mg} /$ day $\times 3$ days $)$ was initiated, followed by oral predonisolone $(50 \mathrm{mg} /$ day $)$ along with prophylactic use of sulfamethoxazole and trimethoprim (480 mg/day). Intermittent hemodialysis therapy was also necessary for uremia and was continued thereafter. Because of her poor renal response to these therapies, a single dose $(500 \mathrm{mg})$ of intravenous cyclophosphamide (IVCY) was performed on day 14 of admission. Because her IgG titer before the use of IVCY was normal $(1,825 \mathrm{mg} / \mathrm{dl})$, we did not use intravenous Ig for prophylaxis. In response to these aggressive immunosuppressive therapies, the autoantibodies titers, including anti-dsDNA IgG, anti-ssDNA IgG, anti-RNP antibody, and MPOANCA, gradually decreased to $14 \mathrm{IU} / \mathrm{ml}, 40 \mathrm{AU} / \mathrm{ml}$, $1,530 \mathrm{IU} / \mathrm{ml}$, and $<10 \mathrm{EU}$, respectively, on day 30 of admission. However, her renal function did not recover despite 4 weeks of these treatments. Because other organs did not seem to be involved under AAV or SLE, and her SLE disease activity seemed to be under control, the doses of oral predonisolone were gradually tapered. Unfortunately, she developed cytomegalovirus infection and candida pneumonia at weeks 5 and 11 of admission, respectively. Although antiviral and antifungal therapies were initiated soon after their diagnoses, she died of sepsis caused by candida pneumonia 4 months after admission (Fig. 3).

\section{Discussion}

Several cases considered to have ANCA-associated GN concurrent with LN have been reported [2-10]. Recently, $\mathrm{Yu}$ et al. [4] reported that approximately $20 \%$ of 152 patients who were classified as LN IV-G had crescentic GN 

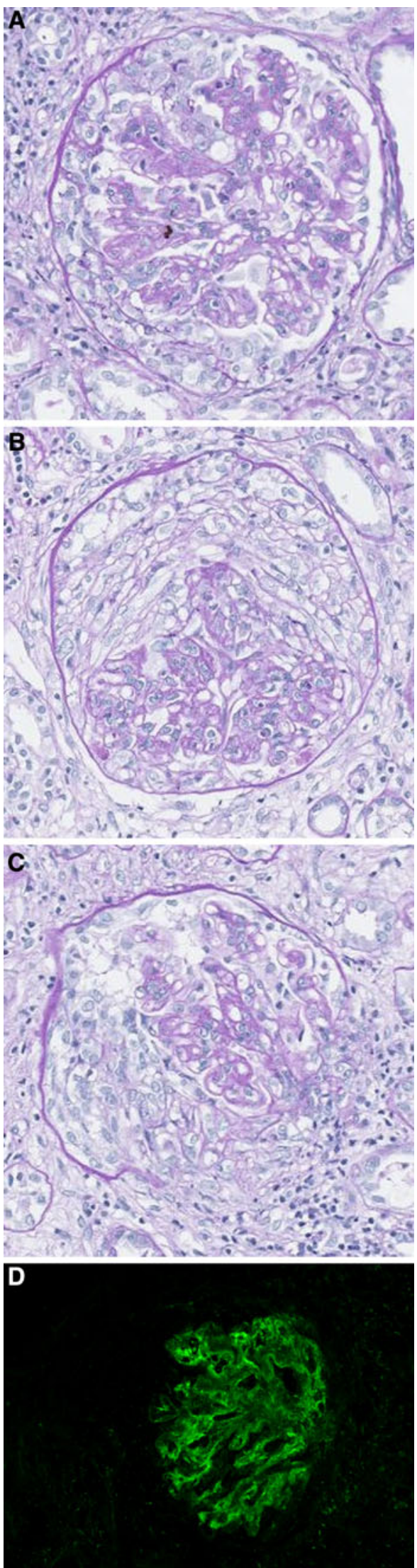

4Fig. 1 Glomeruli showing moderate mesangial cell proliferation with cellular crescents $(\mathbf{a}, \mathbf{b})$. Degeneration of Bowman's capsule is apparent in some glomeruli (c). Direct immunofluorescence showing diffuse staining for $\mathrm{IgG}$ in the basement membrane and the mesangium (d)

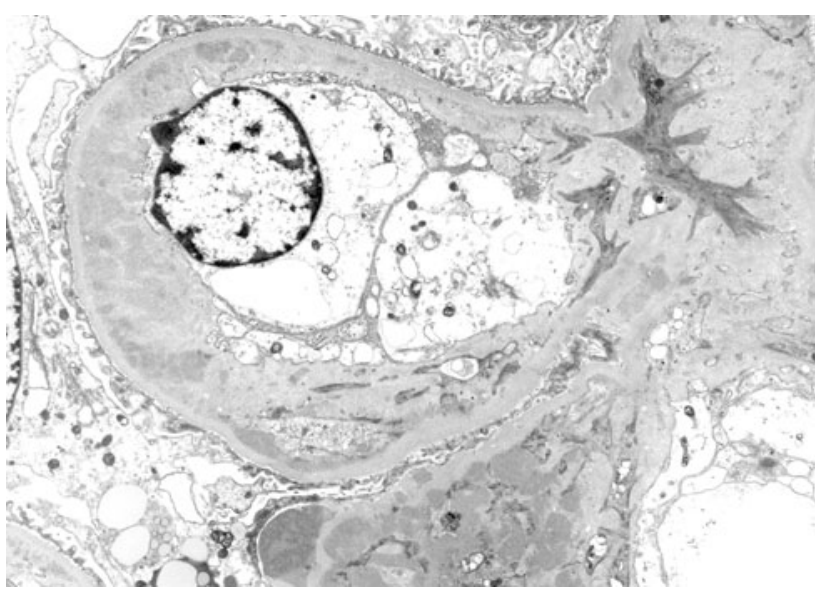

Fig. 2 Electron microscopy showing intramembranous and paramesangial deposits with a few subendothelial deposits

and that approximately $30 \%$ of cases with crescentic LN were seropositive for ANCA in a retrospective study of 327 patients with LN. Those results suggest that crescentic LN seropositive for ANCA is not rare in patients with $\mathrm{LN}$. However, the pathophysiologic link between ANCA-associated GN and LN and the role of ANCA in the development and progression of this condition are not yet fully understood [2-4].

In the present case, the clinical history and laboratory findings, including the appearance of SLE-related autoantibodies and low complement levels, prompted a diagnosis of new-onset SLE with active LN. The renal biopsy revealed active GN. However, several features were observed that are not normally present in LN including extensive crescent formation, no endocapillary proliferation on light microscopy, positive staining for IgG and C3 only on immunofluorescence, and scant endothelial deposits on electron microscopy. Disproportionate crescent formation with no endocapillary proliferation and the presence of very few subendothelial deposits in the presence of MPO-ANCA favored the diagnosis of ANCAassociated crescentic GN. However, our case could not be categorized as having pauci-immune crescentic GN considering the positive immunofluorescent staining. Although "full house" staining for Ig and complement is a typical feature of LN [12], some earlier cases lacked full house staining but were diagnosed with ANCA-associated GN overlapping with LN [2]. Therefore, we diagnosed the 
Fig. 3 Clinical course of the patient after admission. IVCY intravenous cyclophosphamide, $P S L$ predonisolone, $S T$ sulfamethoxazole/trimethoprim, $A B P C / S B T$ ampicillin/ sulbactam, C7-HRP C7 horseradish peroxidase, $C R P$ $\mathrm{C}$-reactive protein, $M P O$ $A N C A$, myeloperoxidasespecific antineutrophil cytoplasmic antibodies

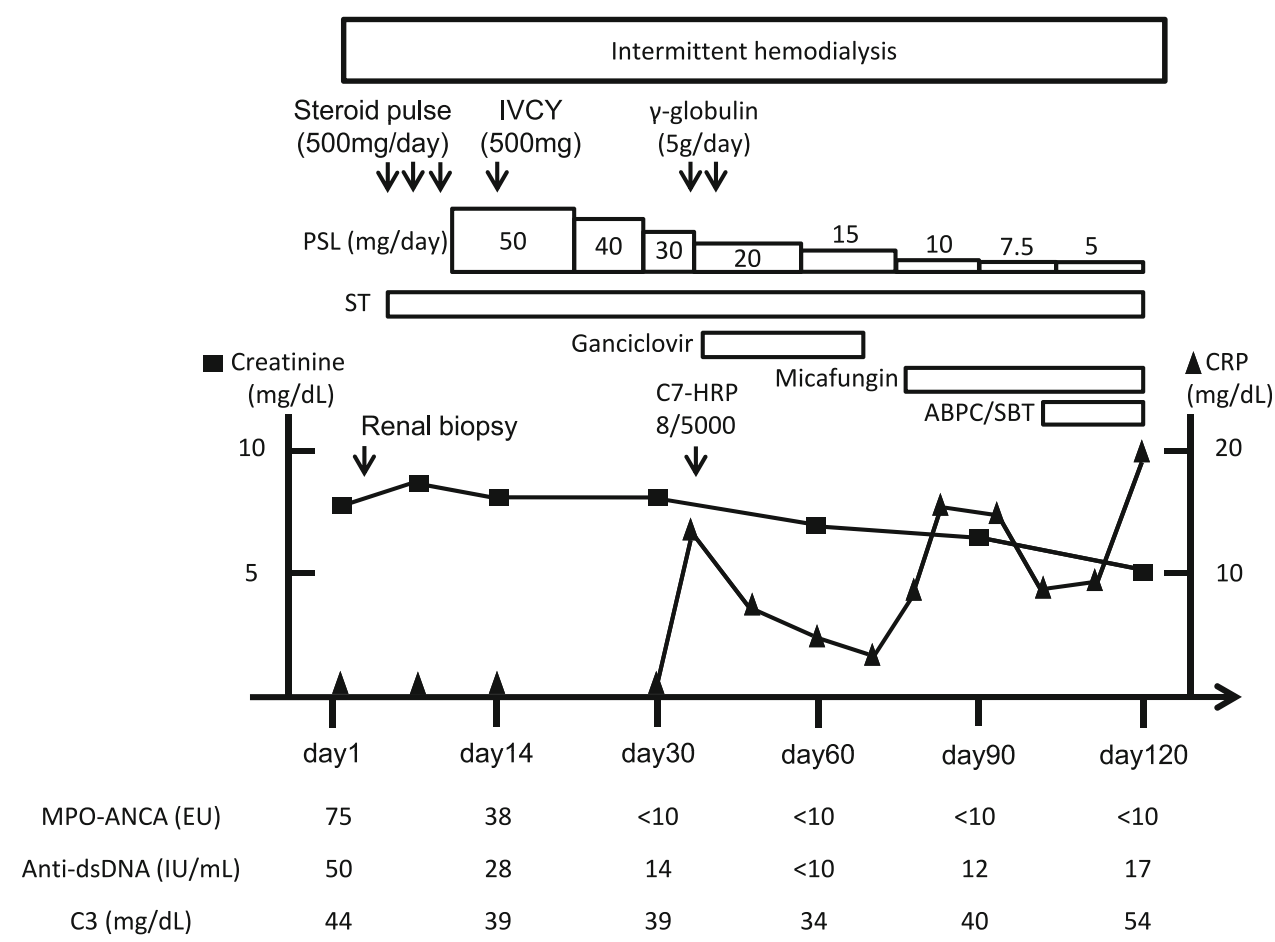

present patient with ANCA-associated crescentic GN overlapping with LN class II.

A particularly interesting feature of our case is the synchronicity of the crescents, which prompted us to speculate that this is involved in the mode of onset in this type of overlapping GN. All of the glomeruli with crescents (21/28 glomeruli, $75 \%)$ had "cellular" crescents. Crescentic GN is categorized immunohistologically into (1) antiGBM GN with linear GBM staining for Ig, which is usually seen in patients with anti-GBM disease; (2) immune complex-mediated crescentic GN with granular staining of glomeruli for Ig or complement, which is usually seen in patients with $\mathrm{LN}$; or (3) pauci-immune crescentic GN, which is usually seen in patients with ANCA-associated GN. A higher frequency of crescent formation (>50\%), as in our patient, is more likely to indicate ANCA-associated GN than LN [13]. However, pauci-immune crescentic GN with ANCA typically presents with an admixture of crescents at different stages, including cellular, fibrocellular, and fibrous crescents [14]. The glomerular lesion synchronicity seen in our case closely resembles the histologic findings of anti-GBM GN. So far, very few reports have referred to the synchronicity of crescents in patients with ANCA-associated GN overlapping with LN. Of the cases reported to date with detailed pathologic findings, most had only cellular $[3,5,7,10]$ or fibrocellular [6] crescents, although one case with different stages of crescents has been reported [9]. The existence of a link between ANCA seropositivity and SLE in the development of GN has been discussed [2-4]. Nasr et al. [2] proposed that one of these two conditions (i.e., ANCA and LN) may provide fertile conditions that encourage the other to develop and that LN might facilitate the development of MPO-ANCA by causing neutrophil degranulation and priming neutrophils to increase surface expression of MPO. The synchronous crescents observed in our patient suggest that ANCAassociated GN, when occurring concurrently with LN, develops more rapidly than the more common ANCAassociated GN without LN and could therefore represent another histologic feature of this overlapping condition. Further accumulation and examination of cases are needed to investigate this hypothesis.

To our knowledge, no cohort study has examined the treatment options or prognosis of this condition. In the treatment of AAV [15], immunosuppressive therapy with IVCY and corticosteroids is mostly used during induction therapy for this overlapping disease. However, their effects and the adverse events (e.g., infection) varied between cases, partly reflecting the comorbidities in each patient [210]. Although our patient required intermittent hemodialysis soon after admission, because of her very active renal histologic status and her severe and acute clinical course, we decided to use immunosuppressive therapies (i.e., MP and IVCY followed by oral corticosteroids). This regimen probably caused the reduction of pathogenic autoantibodies and prevented other organs from being affected by the AAV and SLE disease process. However, this regimen could not recover the patient's renal function. The patient ultimately died because of an infection. The patient's poor response, despite aggressive immunosuppression, may be 
due to delayed initiation of therapy. On the other hand, the poor response may be explained by a poor response of this overlapping condition to conventional immunosuppressive therapy. Yu et al. [4] reported that, in patients with LN IV$\mathrm{G}$ treated with immunosuppressive therapy, the complete remission rate and renal outcome were worse in patients with crescents than in those without crescents. Alternatives to conventional immunosuppressive therapy such as intravenous Ig [6], calcineurin inhibitors [7, 9], and plasma exchange [10] have been reported to achieve partial or complete remission. To achieve remission while minimizing the risk of immunosuppression-related adverse events, further accumulation of clinical evidence documenting the effects of different treatment strategies against this condition is necessary.

In conclusion, the patient had crescentic LN and ANCA seropositivity, which are considered to represent the coexistence of ANCA-associated GN and LN. The patient experienced rapid worsening of her renal function and poor response to aggressive immunosuppressive therapy. The cellular synchronicity of the crescents seen in this case suggests that ANCA-associated GN can develop and progress more rapidly when superimposed on LN. Additionally, this overlapping GN may be resistant to conventional immunosuppressive therapy.

Conflict of interest None.

\section{References}

1. Appel GB, Radhakrishnan J, D'Agati VD. Secondary glomerular disease. In: Brenner BM, editor. Brenner and Rector's the kidney. 8th ed. Philadelphia: WB Saunders; 2008. p. 1067-146.

2. Nasr SH, D'Agati VD, Park HR, Sterman PL, Goyzueta JD, Dressler RM, Hazlett SM, Pursell RN, Caputo C, Markowitz GS. Necrotizing and crescentic lupus nephritis with antineutrophil cytoplasmic antibody seropositivity. Clin J Am Soc Nephrol. 2008;3:682-90.

3. Masani NN, Imbriano LJ, D'Agati VD, Markowitz GS. SLE and rapidly progressive glomerulonephritis. Am J Kidney Dis. 2005;45:950-5.

4. Yu F, Tan Y, Liu G, Wang SX, Zou WZ, Zhao MH. Clinicopathological characteristics and outcomes of patients with crescentic lupus nephritis. Kidney Int. 2009;76:307-17.
5. Marshall S, Dressler R, D’Agati V. Membranous lupus nephritis with antineutrophil cytoplasmic antibody-associated segmental necrotizing and crescentic glomerulonephritis. Am J Kidney Dis. 1997;29:119-24.

6. Arahata $\mathrm{H}$, Migita $\mathrm{K}$, Izumoto $\mathrm{H}$, Miyashita $\mathrm{T}$, Munakata $\mathrm{H}$, Nakamura H, Tominaga M, Origuchi T, Kawabe Y, Hida A, Tagichi T, Eguchi K. Successful treatment of rapidly progressive lupus nephritis associated with anti-MPO antibodies by intravenous immunoglobulins. Clin Rheumatol. 1999;18:77-81.

7. Hirai Y, Iyoda M, Shibata T, Ashikaga E, Hosaka N, Suzuki H, Nagai H, Mukai M, Honda H, Kuroki A, Kitazawa K, Akizawa T. Lupus nephritis associated with positive MPO-ANCA in a patient with underlying autoimmune hemolytic anemia. Clin Exp Nephrol. 2008;12:393-7.

8. Fayaz A, Pirson Y, Cosyns JP, Yango J, Lambert M. Pauciimmune necrotizing and crescentic glomerulonephritis in a patient with systemic lupus erythematosus. Clin Nephrol. 2008;69:290-3.

9. Morimoto S, Watanabe T, Lee S, Amano H, Kanamaru Y, Ohsawa I, Tomino Y, Takasaki Y. Improvement of rapidly progressive lupus nephritis associated MPO-ANCA with tacrolimus. Mod Rheumatol. 2010;20:291-4.

10. Isono M, Araki H, Haitani T, Morita Y, Yasuda M, Kato N, Urasaki K, Tsujimura Y. Diffuse alveolar hemorrhage in lupus nephritis complicated by microscopic polyangiitis. Clin Exp Nephrol. 2011;15:294-8.

11. Weening JJ, D'Agati VD, Schwartz MM, Seshan SV, Alpers CE, Appel GB, Balow JE, Bruijn JA, Cook T, Ferrario F, Fogo AB, Ginzler EM, Hebert L, Hill G, Hill P, Jennette JC, Kong NC, Lesavre P, Lockshin M, Looi LM, Makino H, Moura LA. International Society of Nephrology Working Group on the classification of lupus nephritis; Renal Pathology Society Working Group on the classification of lupus nephritis. The classification of glomerulonephritis in systemic lupus erythematosus revisited. Kidney Int. 2004;65:521-30.

12. D'Agati VD. Renal disease in systemic lupus erythematosus, mixed connective tissue disease, Sjögren's syndrome, and rheumatoid arthritis. In: Jennette JC, Olson JL, Schwartz MM, Silva FG, editors. Heptinstall's pathology of the kidney. 6th ed. Philadelphia: Lippincott Williams and Wilkins; 2007. p. 517-612.

13. Jannette JC. Rapidly progressive crescentic glomerulonephritis. Kidney Int. 2003;63:1164-77.

14. Jannette JC, Nickeleit V. Anti-glomerular basement membrane glomerulonephritis and Goodpasture's syndrome. In: Jennette JC, Olson JL, Schwartz MM, Silva FG, editors. Heptinstall's pathology of the kidney. 6th ed. Philadelphia: Lippincott Williams and Wilkins; 2007. p. 613-41.

15. Bosch X, Guilabert A, Espinosa G, Mirapeix E. Treatment of antineutrophil cytoplasmic antibody-associated vasculitis: a systematic review. JAMA. 2007;298:655-69. 